\title{
ON METRIC SPACES WITH UNIFORM NORMAL STRUCTURE
}

\author{
M. A. KHAMSI
}

(Communicated by Burgess J. Davis)

\begin{abstract}
In this work, we prove that metric spaces with uniform normal structure have a kind of intersection property, which is equivalent to reflexivity in Banach spaces.
\end{abstract}

\section{INTRODUCTION}

It has always been tempting to generalize certain existence fixed point theorems to metric spaces. In 1969, Kijima and Takahashi [3] gave a metric formulation of Kirk's theorem [4]. But their definition of convex metric spaces is rather restraining. In our opinion, many results with respect to the fixed point property in metric spaces, were developed after Penot's formulation [8]. The compactness of the convexity structure which appears in this formulation expresses the weak compactness, or more precisely, the reflexivity in the case of Banach spaces.

In this paper we generalize Maluta's theorem [6] to metric spaces. Recall that the proof given by Maluta is based on a characterization of reflexive Banach spaces described by Mil'man and Mil'man [7]. This characterization uses the linear structure and we do not know how the proof could be reproduced in metric spaces.

\section{DEFINITIONS AND BASIC RESULTS}

In all the following in this work $(M, d)$ will be a metric space.

Definition 1. Let $\mathscr{F}$ be a nonempty family of subsets of $M$. We say that $\mathscr{F}$ defines a convexity structure on $M$ if and only if $\mathscr{F}$ is stable by intersection.

In this work, we will always assume that $\mathscr{F}$ contains the ball, and, therefore, we will not recall it.

Definition 2. We say that $\mathscr{F}$ has the property $(R)$ if and only if for any decreasing sequence $\left(C_{n}\right)$ of closed bounded nonempty subsets of $M$, with $C_{n} \in \mathscr{F}$, has a nonempty intersection.

Received by the editors October 20, 1988.

1980 Mathematics Subject Classification (1985 Revision). Primary 47H10, 47H09.

Key words and phrases. Nonexpansive mappings, fixed point property, uniform normal structure. 
Remark 3. In [8], Penot defined a notion of compactness of a convexity structure. Here we will avoid using the term "compact", since it creates some confusion with the topological compactness. Then we will say that $\mathscr{F}$ has the finite intersection property if and only if any family $\left(C_{\alpha}\right)_{\alpha \in \Gamma}$ of elements of $\mathscr{F}$ has a nonempty intersection provided $\cap_{\alpha \in I} C_{\alpha} \neq \varnothing$ for any finite subset $I$ of $\Gamma$.

Example 4. (i) Let $C$ be a closed bounded convex subset of Banach space $X$. Consider the family $\mathscr{F}$ of closed convex subsets of $C$. Then $\mathscr{F}$ defines a convexity structure on $C$, and $\mathscr{F}$ has the property $(R)$ if and only if $C$ is weakly compact.

(ii) An admissible subset of $M$ (e.g.[1]) is any intersection of closed balls. Let us denote the family of admissible subsets of $M$ by $\mathscr{A}(M)$. It is obvious that $\mathscr{A}(M)$ defines a convexity structure on $M$. In this work any other convexity structure $\mathscr{F}$ on $M$, contains $\mathscr{A}(M)$.

Definition 5. Let $N$ be a bounded subset of $M$.

(i) Define:

$$
\begin{aligned}
r(x, N) & =\sup \{d(x, y) ; y \in N\} \text { for } x \in M . \\
\delta(N) & =\sup \{r(x, N) ; x \in N\} . \\
R(N) & =\inf \{r(x, N) ; x \in N\} . \\
\mathscr{C}(N) & =\{x \in N ; R(N)=r(x, N)\} .
\end{aligned}
$$

(ii) We will say that $x \in N$ is a diametral point if $r(x, N)=\delta(N)$, and $N$ is a dimetral set if any elements of $N$ is a diametral point.

Now, we are able to define the normal and uniform normal structure in metric spaces.

Definition 6. We say that $M$ has normal structure [resp. uniform normal structure] if there exists a convexity structure $\mathscr{F}$ on $M$ such that: $R(A)<\delta(A)$ [resp. $R(A) \leq c \delta(A)$ for a fixed constant $c \in(0,1)$ ] for any nonempty $A \in \mathscr{F}$, which is bounded and not reduced to a single point. We will also say that $\mathscr{F}$ is normal [resp. uniformly normal]. tion.

Since our work uses the fixed point property, let us give the following defini-

Definition 7. A mapping $T: M \rightarrow M$ is said to be nonexpansive if $d(T x, T y)$ $\leq d(x, y)$ for all $x, y \in M$. The fixed point set of $T$ will be denoted by $\operatorname{Fix}(T)$. We say that $M$ has the fixed point property if any nonexpansive mapping defined on $M$ has a fixed point.

The analogue of Kirk's theorem [4] in metric spaces can be stated by the following:

Theorem 8. Let $M$ be a bounded metric space. Assume that there exists a convexity structure $\mathscr{F}$ on $M$, which is normal and has the finite-intersection property. Then $M$ has the fixed point property. 
Remark 9. Kirk noticed in [5], that the finite-intersection property assumption can be dropped in Theorem 8, provided that $F$ has a kind of sequential finite intersection property. It is easy to see that this sequential finite-intersection property is equivalent to the property $(R)$.

\section{ON THE PROPERTY $(R)$}

Before we give our first result, let us recall that Gillespie and Williams [2] proved that a closed bounded nonempty convex subset of Banach space, which has uniform normal structure, has the fixed point property.

Theorem 10. Let $(M, d)$ be a complete bounded metric space. Assume that $M$ has uniform normal structure. Then $M$ has the fixed point property.

Proof. Kirk proved in [5], the following lemma, which can also be derived from the Gillespie and William's proof.

Lemma 11. Let $M$ be a metric space. Consider $T: M \rightarrow M$, a nonexpansive mapping. Then any nonempty admissible subset $A$ of $M$, which is $T$-invariant, contains a nonempty admissible subset $A_{0}$ of $M$ which is $T$-invariant and verifies:

$$
\delta\left(A_{0}\right) \leq \frac{\delta(A)+R(A)}{2} .
$$

Using the Lemma 11, the proof of Theorem 10 is immediate. Indeed, let $T: M \rightarrow M$ be a nonexpansive mapping. Since $M$ has uniform normal structure, there exists $c \in), 1$ ( such that $R(A) \leq c \delta(A)$ for any admissible subset $A$ of $M$. Since $M$ is bounded, we can apply the Lemma 11 to insure the existence of a decreasing sequence $\left(A_{n}\right)$ of nonempty admissible subsets of $M$ such that $A_{n}$ is $T$-invariant and $\delta\left(A_{n+1}\right) \leq\left(R\left(A_{n}\right)+\delta\left(A_{n}\right)\right) / 2$. This implies that:

$$
\delta\left(A_{n}\right) \leq\left(\frac{1+c}{2}\right)^{n} \delta(M)
$$

Therefore $\cap A_{n} \neq \varnothing$, since $M$ is complete.

(*) implies that $\cap A_{n}$ is reduced to a single point, which is fixed by $T$.

To prove the analogue of Maluta's result [6] in metric spaces, we will need the following result.

Proposition 12. Let $M$ be a bounded metric space, and $\left(M_{\alpha}\right)_{\alpha \in \Gamma}$ be a family of nonempty subset of $M$. Assume that each $\mathscr{A}\left(M_{\alpha}\right)$ has uniform normal structure and $\left.c=\sup c_{\alpha} \in\right) 0,1\left(\right.$, where $c_{\alpha}$ is the constant which appears in Definition 6. Consider the metric $M_{\infty}=\prod^{\alpha} M_{\alpha}$ provided with the sup distance. Then $\mathscr{A}\left(M_{\infty}\right)$ has uniform normal structure.

Proof. Let $A$ be an admissible subset of $M_{\infty}$. Then $A=\cap_{i \in I} B\left(a_{i}, r_{i}\right)$. It is clear that $B\left(a_{i}, r_{i}\right)=\prod_{\alpha \in \Gamma} B^{\alpha}\left(a_{i}^{\alpha}, r_{i}\right)$ where $a_{i}^{\alpha}$ is the $\alpha$ th-component of $a_{i}$, and $B^{\alpha}\left(a_{i}^{\alpha}, r_{i}\right)$ is the closed ball centered at $a_{i}^{\alpha}$ in $M_{\alpha}$. Therefore $A=\prod_{\alpha \in \Gamma} A_{\alpha}$, where $A_{\alpha}=\cap_{i \in I} B^{\alpha}\left(a_{i}^{\alpha}, r_{i}\right)$. Our assumption on $(\mathscr{A}(M))_{\alpha \in \Gamma}$ 
implies that $R\left(A_{\alpha}\right) \leq c \delta\left(A_{\alpha}\right)$ for all $\alpha \in \Gamma$. But, $\delta(A)=\sup _{\alpha \in \Gamma} \delta\left(A_{\alpha}\right)$ and $R(A) \leq \sup _{\alpha \in \Gamma} R\left(A_{\alpha}\right)$. We deduce that $R(A) \leq c \delta(A)$. Therefore $\mathscr{A}\left(M_{\infty}\right)$ has uniform normal structure.

Theorem 13. Let $M$ be a complete bounded metric space. Let $\mathscr{F}$ be a convexity structure on $M$ which has uniform normal structure. Then $\mathscr{F}$ has the property $(R)$.

Proof. Let $\left(C_{n}\right)$ be a decreasing sequence of nonempty closed bounded subsets of $M$, with $C_{n} \in \mathscr{F}$. Put $C_{\infty}=\prod_{n} C_{n}$ provided with the sup distance. By Proposition 12, we deduce that $\mathscr{A}\left(C_{\infty}\right)$ has uniform normal structure. Since $M$ is complete and each $C_{n}$ is closed, $C_{\infty}$ is a complete metric space. Therefore we deduce from Theorem 10 that $C_{\infty}$ has the fixed point property. Consider the shift $T: C_{\infty} \rightarrow C_{\infty}$ defined by $T\left(x_{n}\right)=\left(x_{n+1}\right)$. Obviously $T$ is nonexpansive, and therefore $T$ has a nonempty fixed point set. Let $\left(x_{n}\right) \in \operatorname{Fix}(T)$, by definition of $T,\left(x_{n}\right)$ is a constant sequence. Consequently $x_{0}=x_{n} \in C_{n}$ for all $n \in \mathbf{N}$. This implies that $\cap C_{n} \neq \varnothing$.

\section{ACKNOWLEDGMENT}

I would like to thank J. B. Baillon and R. Sine with whom I had fruitful discussions regarding this work.

\section{REFERENCES}

1. N. Dunford and J. T. Schwartz, Linear operator, Part 1, Interscience, New York, 1958.

2. A. A. Gillespie and B. B. Williams, Fixed point theorem for nonexpansive mapping on Banach spaces with uniformly normal structure, Appl. Anal. 9 (1979), 121-124.

3. Y. Kijima and W. Takahashi, A fixed point theorem for nonexpansive mappings in metric spaces, Kodai, Math. Sem. Rep. 21 (1969), 326-330.

4. W. A. Kirk, A fixed point theorem for mappings which do not increase distance, Am. Math. Mon. 72 (1969), 1004-1006.

5. _. Nonexpansive mappings in metric and Banach spaces, Estratto Dai (Rendiconti del Seminario Matematico e Fisico di Milano) Vol L1 (1981), 133-144.

6. E. Maluta, Uniformly normal structure and related coefficients, Pacific J. Math. vol. 111 \#2 (1984), 357-369.

7. D. P. Mil'man and V. D. Mil'man, The geometry of nested families with empty intersectionstructure of the unit ball of a nonreflexive space, Amer. Math. Soc. Trans. (2) 85 (1966), 233-243.

8. J. P. Penot, Fixed point theorem without convexity, Analyse non convexe (1977, Pau) Bull. Soc. Math. France, Memoire 60 (1979), 129-152.

University of SOUthern California, Los ANGeles, California 90089-0782 